\title{
Expression of Ki-67, p53 and p63 proteins in keratocyst odontogenic tumours: an immunohistochemical study
}

\author{
Clarissa Araújo Silva Gurgel · Eduardo Antônio Gonçalves Ramos • \\ Roberto Almeida Azevedo - Viviane Almeida Sarmento • \\ Ana Maria da Silva Carvalho · Jean Nunes dos Santos
}

Received: 2 October 2007/ Accepted: 23 January 2008/Published online: 7 February 2008

(C) Springer Science+Business Media B.V. 2008

\begin{abstract}
Aim To investigate the immunohistochemical expression of Ki-67, p53 and p63 in Keratocyst Odontogenic Tumours (KOTs) in order to contribute to the biological profile of this tumor. Methods Immunohistochemical technique was performed using the EnVision ${ }^{\mathrm{TM}}$ System in 37 cases of KOTs. Results Ki-67- and p53immunostained cells were mainly located in the suprabasal layers. p63-positive cells were found throughout the lining cystic epithelium. No difference in the immunostaining for these proteins was observed between primary and recurrent KOTs (Ki-67: $P=0.5591 ;$ p53: $\quad P=0.9847$; p63: $P=0.9127)$, or between KOTs associated with Nevoid Basal Cell Carcinoma Syndrome (NBCCS) and sporadic KOTs (Ki-67: $P=0.7013$; p53: $P=0.3197$; p63: $P=0.2427$ ). Conclusions It is possible that biological behavior of KOTs may be related to suprabasal proliferative compartment in the cystic epithelium as observed by
\end{abstract}

C. A. S. Gurgel - E. A. G. Ramos - A. M. da Silva Carvalho Laboratory of Histopathology of the Gonçalo Moniz Research Center-Oswaldo Cruz Foundation, Salvador, Bahia, Brazil

R. A. Azevedo

Division of Oral and Maxillofacial Surgery, School of Dentistry of the Federal University of Bahia, Salvador, Bahia, Brazil

\section{A. Sarmento}

Division of Oral Radiology, School of Dentistry of the Federal University of Bahia, Salvador, Bahia, Brazil

J. N. dos Santos

Laboratory of Oral Surgical Pathology of School of Dentistry of the Federal University of Bahia, Salvador, Bahia, Brazil

J. N. dos Santos $(\bowtie)$

Faculdade de Odontologia-UFBA, Avenida Araujo Pinho, 62, Canela, Salvador, Bahia 40110-150, Brazil

e-mail: jeanunes@ufba.br high levels of Ki-67, p53 and p63. In addition, p63 immunostaining may represent immaturity of keratinocytes in KOTs, and suggests that this protein may participate in the regulation of epithelial cell differentiation. Taken together, these data may favor tumorigenesis on KOTs.

Keywords Odontogenic cysts $\cdot$ Keratocyst odontogenic tumour $\cdot$ Cell proliferation $\cdot \mathrm{Ki}-67 \cdot \mathrm{p} 53 \cdot \mathrm{p} 63$

\section{Introduction}

Keratocyst Odontogenic Tumours (KOTs) are lesions affecting the jawbones, especially in young adults, whose biological behavior differs from that of other odontogenic cysts. Classically, KOTs are considered to be developmental cysts arising from remnants of the dental lamina (Kolar et al. 2006). However, their aggressive clinical behavior, associated with a high rate of recurrence, infiltrative growth and association with Nevoid Basal Cell Carcinoma Syndrome (NBCCS) suggests a neoplastic origin of these lesions (Shear 2002a, b; Agaram et al. 2004; Kolar et al. 2006). KOTs are frequently found in the posterior region of the mandible (Oda et al. 2000; Stoelinga 2001; Giuliani et al. 2006).

The growth mechanism of KOTs has been investigated by different researchers, especially the proliferative potential of the epithelial lining (Shear 2002b; Kim et al. 2003; Agaram et al. 2004; Thosaporn et al. 2004) including proteins involved in the cell cycle (Kimi et al. 2000; Kichi et al. 2005). The Ki-67 antigen is a non-histone protein with a molecular weight ranging from 345 to $395 \mathrm{kDa}$ (Gerdes et al. 1991; McCormick et al. 1993). It is encoded by a single gene on chromosome 10 (McCormick et al. 1993), being part of the nuclear matrix during 
interphase and binding to the chromosome during mitosis (Verheijen et al. 1989). In KOTs, Ki-67 has been found to be overexpressed (Kim et al. 2003; Kaplan and Hirshberg 2004; Kichi et al. 2005). The p53 gene encodes a protein of the same name with a molecular weight of $53 \mathrm{kDa}$, whose functions are related to the control of cell proliferation and apoptosis (Levine et al. 1991; Lane 1992). Despite the continuous transcription of this gene, the half-life of the p53 protein is short and the protein does not reach high concentrations in non-lesioned cells (Morgunkova 2005). KOTs express high levels of p53 (Ogden et al. 1992; Slootweg 1995; Lo Muzio et al. 1999; Kichi et al. 2005). The p63 gene encodes at least six isoforms which are subdivided into two groups according to the presence or absence of a transcriptional activation domain: the TAp63 isoforms contain the transcriptional activation domain and are involved in apoptosis, whereas the $\Delta \mathrm{Np} 63$ isoforms, which do not contain this domain, participate in the cell proliferation (Yang et al. 1998; Little and Jochemsen 2002; Barbiere and Pietenpol 2005). This protein, a p53 homologue, is essential for regenerative proliferation in epithelial development, however, p63 may act as an oncogen (Yang et al. 1998; Yang et al. 2002; Mills 2006).

Regarding the local invasiveness of KOTs, several authors have found expression of Ki-67 and p53 in these lesions, indicating a key role in the growth and proliferative activity (Slootweg 1995; Kichi et al. 2005; GonzalezMoles et al. 2006). However, to our knowledge, little is known about the distribution of p63 in KOTs and its association with $\mathrm{Ki}-67$ and p53. Thus, we performed this study to evaluate the immunoreactivity of Ki-67, p53 and p63 in epithelial cells lining keratocysts in order to contribute to the biological profile of this tumor.

\section{Material and methods}

After Institutional Ethics Committee approval, 37 cases of KOTs obtained from excisional biopsy were selected from the files of the Laboratory of Oral Surgical Pathology, School of Dentistry of the Federal University of Bahia (FOUFBA, Brazil). They were analyzed and selected based on the anatomopathological reports.

For morphological analysis, material fixed in formalin and embedded in paraffin was cut $4 \mu \mathrm{m}$ thick sections. The Hematoxylin/Eosin-stained slides of each case were submitted to a new histological exam by light microscopy in order to assess the morphological aspects representative of each lesion.

Immunohistochemistry was performed on formalinfixed and paraffin-embedded $3 \mu \mathrm{m}$ thick sections. The tissue sections were deparaffined, and for antigen retrieval, conditions included sections boiled in citrate monohydrate solution for $30 \mathrm{~min}, \mathrm{pH} 6.2$, in humid heat at $96^{\circ} \mathrm{C}$. The reactions were treated with peroxidase block solution (EnVision ${ }^{\mathrm{TM}}$ System) for $10 \mathrm{~min}$ to quench endogenous peroxidase activity. Monoclonal antibodies against Ki-67 (Clone KIS5, 1:100 dilution, DAKO Cytomation, Glostrup, Denmark), p53 (Clone DO-7, 1:50 dilution, DAKO Cytomation, Glostrup, Denmark), p63 (Clone 4A4, 1:150 dilution, DAKO Cytomation, Glostrup, Denmark) were applied with EnVision ${ }^{\mathrm{TM}}$ System (DAKO Corporation, Carpinteria, CA, USA).

The tissue sections were then exposed to antibodies for $40 \mathrm{~min}$ at room temperature using an antibody diluent with background reducing components (DAKO Corporation, Carpinteria, USA). Immunohistochemical reactions were developed with diaminobenzidine as the chromogenic peroxidase substrate and the slides were counterstained with Harris Hematoxylin. Oral squamous cell carcinomas tissues served as positive controls. Negative controls included replacement of the primary antibody with nonimmune bovin serum albumin.

For the analysis of immunostaining, the slides were examined with a light microscope coupled to a digital camera system (Axiocam HRP, Zeiss, Germany, 2004) at a final magnification of $400 \times$. For each case a minimum of 1000 nuclei located in the basal and suprabasal layers were counted in up to 10 consecutive microscopic fields per case using the Image Tool 2.0 software (UTHSCSA, Texas University, USA, 1996).

The immunoreactive cells were evaluated semiquantitatively using the following scores: negative (0 to $5 \%$ of cells immunostained), +1 (6 to $25 \%$ immunostained), +2 (26 to $50 \%)$, and $+3(>50 \%)$. In addition, clinical features as recurrence and association with NBCCS also were compared.

The differences between groups were tested using statistically Mann-Whitney. All statistical calculations were performed with BIOESTAT 3.0 program (Sociedade Civil Mamirauá, MCT-CNPq, Conservation International, Brazil, 2003). The level of significance was set at $5 \%$.

\section{Results}

Thirty seven lesions diagnosed as KOTs between 2003 and 2006 were selected in this study, including nine lesions diagnosed in five patients with NBCCS. Of all KOTs, 14 (46.67\%) were recurrent lesions, during a mean follow-up of 24 months. Histopathologically, all KOTs showed a thin cystic wall exhibiting an epithelial lining of uniform thickness as well as parakeratinized and corrugated surface. Twenty-six cases displayed significant inflammation.

Ki-67 immunostaining was detected in 36 (97.3\%) cases, mainly in the suprabasal layers of the cystic 
epithelium. In one case, all cells were negative for this antibody. Immunostaining was scored as +1 in most cases $(n=18,48.65 \%)$. p53 immunostaining was observed in 34 $(91.9 \%)$ cases, mainly in the suprabasal layers. p53 immunostaining was scored as +3 in $51.35 \%(n=19)$ of KOTs. p63 was expressed in all cases studied $(n=37$, $100.0 \%)$ and involved all epithelial layers. Immunostaining for this marker was scored as +3 in all KOTs analyzed (Tables 1 and 2, Fig. 1a-f).

No significant difference in the expression of Ki-67, p53 and p63 was observed between groups of lesions associated with NBCCS $(n=9)$ and sporadic KOTs $(n=28)$ (Table 3$)$ or between primary $(n=23)$ and recurrent lesions $(n=14)$ (Table 4).

\section{Discussion}

$\mathrm{Ki}-67$ has been used as excellent marker of cell proliferation (Li et al. 1995; Slootweg 1995; Lo Muzio et al. 1999; De Paula et al. 2000; Kaplan and Hirshberg 2004). Its level increases durant the $\mathrm{S}$ phase but MIB-1 recognizes Ki-67 antigen in all cell cycle. Studies comparing KOTs and dentigerous cysts have demonstrated a greater proliferative potential of the epithelium of KOTs comparable to that of ameloblastomas ( $\mathrm{Li}$ et al. 1995; Thosaporn et al. 2004). In this study, cells immunostained for the anti-Ki-67 antibody were predominantly found in the suprabasal layer. In syndromic patients, Ki-67 was expressed in all cell layers of the cystic epithelium. These results suggest a dysregulation of the cell cycle and agree with studies indicating the presence of a suprabasal proliferative compartment in KOTs (Lo Muzio et al. 1999; Shear 2002c; Kim et al.

Table 1 Distribution of the number of lesions positive for the Ki-67, p53 and p63 antibodies according to mean score

\begin{tabular}{lccc}
\hline Score & Ki-67 $n^{\mathrm{a}}(\%)$ & $\mathrm{p} 53 n(\%)$ & $\mathrm{p} 63 n(\%)$ \\
\hline- & $5(13.5)$ & $3(8.11)$ & $0(0)$ \\
+1 & $18(48.65)$ & $4(10.81)$ & $0(0)$ \\
+2 & $13(35.13)$ & $11(29.73)$ & $0(0)$ \\
+3 & $1(2.72)$ & $19(51.35)$ & $37(100)$ \\
\hline
\end{tabular}

${ }^{a}$ Four of the five lesions classified as negative for Ki-67 presented less than $5 \%$ of immunostained cells, and one case presented all cells negative for this protein
2003; Kaplan and Hirshberg 2004; Kichi et al. 2005). Although the distribution of Ki-67 was more uniform in the cystic epithelium of syndromic patients, no significant difference in immunostaining could be demonstrated between lesions associated with NBCCS and sporadic KOTs $(P=0.7013)$. Similar results have been reported by $\mathrm{Li}$ et al. (1995). In contrast, Lo Muzio et al. (1999) suggested that the higher expression of cell proliferation markers in syndromic patients reflects a more aggressive behavior of these lesions, especially in terms of a greater tendency to recurrence.

Also regarding the proliferative activity of the lining epithelium of KOTs, some investigators have analyzed the expression of p53 (Ogden 1992; Lombardi et al. 1995; Slootweg 1995; Kichi et al. 2005). p53 is a tumor suppressor gene effective at the G1 phase of cell cycle, which participates in the growth arrest, initiates repair, or induces apoptosis (Levine et al. 1991, Lane 1992; Morgunkova 2005). The biological mechanism associated with the expression of this protein in the epithelium of KOTs has not been defined, but the aggressive behavior and high recurrence rate of these lesions might be related to the immunoexpression of this protein (Lombardi et al. 1995) In the present study, 34 (91.9\%) cases were positive for p53. Most of them presenting a score of $+3(n=19$, $51.35 \%$ ), and identified in the suprabasal layers of the cystic epithelium as also demonstrated by Lombardi et al. (1995), Slootweg (1995), Li et al. (1996), Piattelli et al. (2001), Kichi et al. (2005) and Kolar et al. (2006). No significant difference in p53 immunostaining was observed between the lesions associated with NBCCS and sporadic KOTs $(P=0.3195)$, or between primary and recurrent lesions $(P=0.9847)$. These results were similar to those found by Lombardi et al. (1995), Li et al. (1996) and Piattelli et al. (2001). However, in the present study the percentage of p53-positive KOTs was higher than those reported by Ogden et al. (1992), Lombardi et al. (1995) and Piattelli et al. (2001). This finding might be attributed to the use of the immunohistochemical method.

Although we did not observe any difference in p53 immunostaining between KOTs associated with NBCCS and sporadic KOTs, the distribution of positive cells among epithelial layers was more homogeneous in the first group. This finding is similar to the results of Lo Muzio et al. (2005). Immunodetection of this protein seems to be

Table 2 Distribution of positive cells for Ki-67, p53 and p63 in basal and suprabasal cells

\begin{tabular}{lclllll}
\hline & Ki-67 & $P$ & p53 & $P$ & p63 & $P$ \\
\hline Basal cells & $49.4 \pm 44$ & $P<0.0001$ & $205.8 \pm 116.2$ & $P<0.0001$ & $371.8 \pm 138.4$ & $P<0.0001$ \\
Suprabasal cells & $154.6 \pm 127.6$ & & $357.0 \pm 165.3$ & & $585.1 \pm 214.8$ \\
\hline
\end{tabular}

Mann-Whitney Test, Values are means \pm standard deviation 
Fig. 1 Immunohistochemical staining of Keratocyst Odontogenic Tumours.

(a) +1 score in a non-syndromic patient exhibiting Ki-67 expression predominantly in suprabasal cells (EnVision ${ }^{\mathrm{TM}}$ System, approximately $400 \times$ ). (b) +3 score in a NCBBS patient exhibiting Ki-67 expression predominantly in suprabasal cells (EnVision ${ }^{\mathrm{TM}}$ System, approximately $400 \times)$. (c) +2 score in a non-syndromic patient exhibiting p53 expression predominantly in suprabasal cells (EnVision ${ }^{\mathrm{TM}}$ System, approximately $400 \times$ ). (d) +3 score in a NCBBS patient exhibiting p53 expression predominantly in suprabasal cells (approximately $400 \times$ ). (e) +3 score in a NCBBS patient showing p63 expression in basal and suprabasal cells

EnVision $^{\mathrm{TM}}$ System, approximately $400 \times$ ). (f) +3 score non- syndromic patient showing p63 expression in basal and suprabasal cells $\left(\right.$ EnVision $^{\mathrm{TM}}$ System, approximately $400 \times$ )
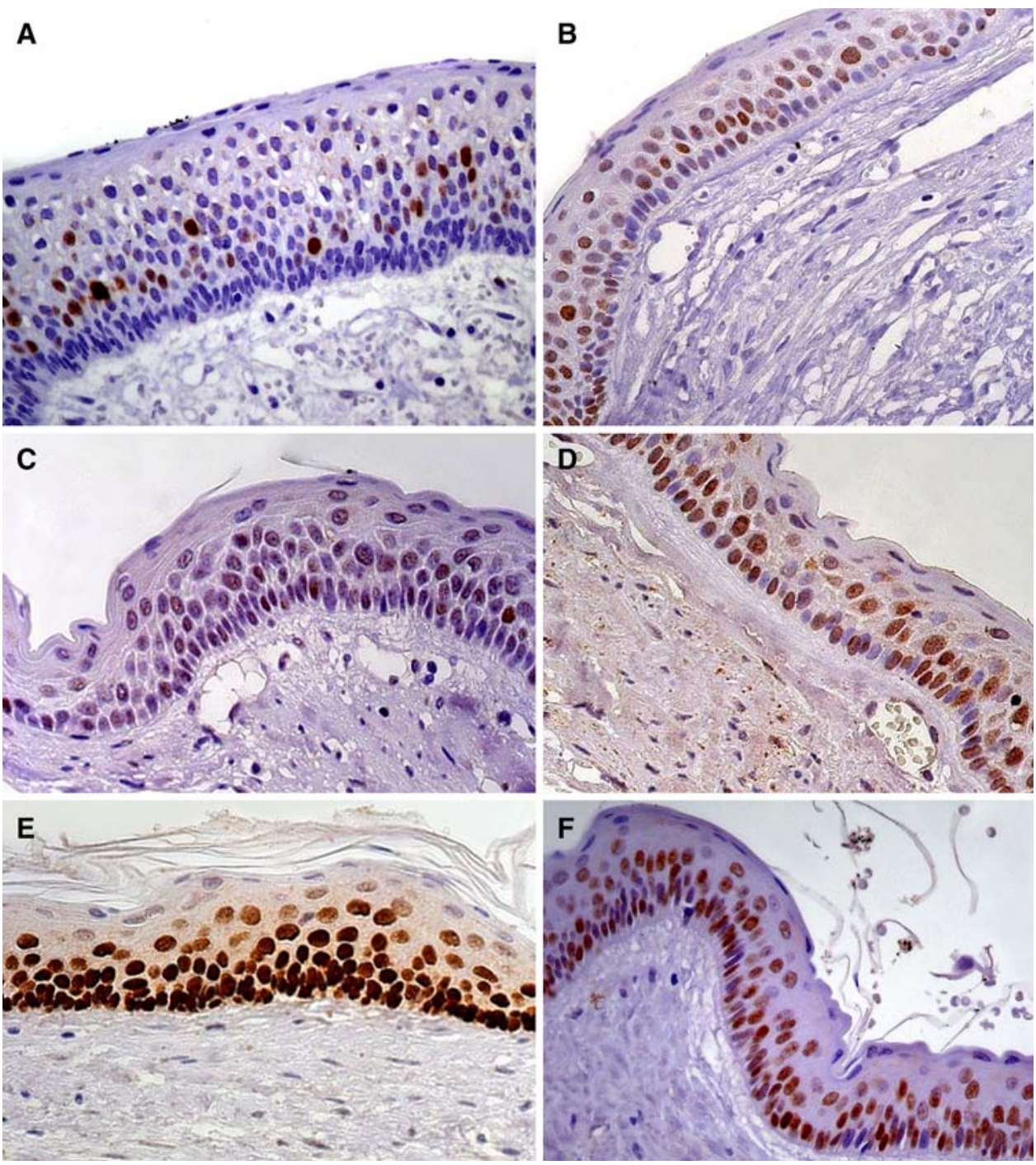

Table 3 Median number of cells immonostained for Ki-67, p53 and p63 between KOTs associated with NBCCS and sporadic OKs

\begin{tabular}{lllllll}
\hline & Ki-67 & $P$ & p53 & $P$ & p63 & $P$ \\
\hline NBCCS $(n=9)$ & $150.3 \pm 76.3$ & $P=0.7013$ & $150.3 \pm 76.3$ & $P=0.3195$ & $627.1 \pm 190$ & $P=0.2427$ \\
Sporadic OKs $(n=28)$ & $156.7 \pm 141.4$ & & $156.7 \pm 141.4$ & & $571.6 \pm 223.7$ & \\
\hline
\end{tabular}

Mann-Whitney Test, Values are means \pm standard deviation

Table 4 Difference in the immunostaining for Ki-67, p53 and p63 between primary and recurrent KOTs

\begin{tabular}{lllllcc}
\hline & Ki-67 & $P$ & p53 & $P$ & p63 & $P$ \\
\hline Primary Oks $(n=23)$ & $235.5 \pm 222.2$ & $P=0.5591$ & $557.2 \pm 284.4$ & $P=0.9847$ & $1008.0 \pm 311.6$ & $P=0.9127$ \\
Recurrent OKs $(n=14)$ & $177.6 \pm 124.8$ & & $561.8 \pm 318.7$ & & $971.0 \pm 303.3$ \\
\hline
\end{tabular}

Mann-Whitney Test, Values are means \pm standard deviation

related to the stabilization of $\mathrm{p} 53$ product, a fact reflecting cell cycle regulation in favor of proliferation (Slootweg 1995; Piattelli et al. 2001; Shear 2002b), indicating an intrinsic growth potential of the KCOT epithelium. Moreover, we found similar distribution between Ki-67 and p53positive cells. 
It is possible that the accumulation of p53 in KOTs can not be attributed to gene mutation with stabilization of this protein, but it may be due to an accumulation of normal p53 protein ( $\mathrm{Li}$ et al. 1996; Gonzalez-Moles et al. 2006). This explanation might be attributed to $\mathrm{mdm} 2$ defects as a response in different situations (Save et al. 1998). However, it is important to state that Agaram et al. (2004) and Henley et al. (2005) have demonstrated lost of heterozigosity in TPp53 gene in KOTs. Further studies are needed to clarify this matter.

Yang et al. (1999) and Little and Jochemsen (2002) reported the existence of different isoforms of p63, TAp63 and $\triangle \mathrm{Np} 63$, that are involved in apoptosis and cell proliferation, respectively. Clone 4A4 used here in the immunohistochemical reactions recognizes both the TAp63 and $\Delta$ Np63 isoforms. However, Nylander et al. (2002) observed that this clone only labels the non-transactivated isoforms in stratified squamous epithelia. In agreement with this hypothesis, Parsa et al. (1999) and De Laurenzi et al. (2000) only detected $\Delta$ Np63 RNA in human keratinocytes.

Studies using an anti-p63 antibody for the investigation of odontogenic lesions are scarce and only two reports investigating the expression of this protein in KOTs are available. In 2005, Lo Muzio et al. (2005) published the first study describing the immunostaining of p63 in KOTs. These authors used a clone similar to ours, however, that study included an orthokeratinized variant, a distinct and less aggressive disease (Thosaporn et al. 2004; Philipsen 2005). In 2006, Foschini et al. (2006) reported a more homogenous and superficial distribution of p63 in recurrent OKs, using a polyclonal antibody (antip-40). In the present study, p63 immunostaining was demostrated in all 37 KOTs analyzed (score +3 ) and involved all epithelial layers. The expression of this protein was not associated with the clinical features investigated, such as recurrence $(P=0.9127)$ or NBCCS $(P=0.2427)$. Lo Muzio et al. (2005) observed intense p63 positivity in epithelial cells of KOTs, including more superficial cells, and in $64 \%$ of cases this staining was found to be intense in up to $50 \%$ of cells of the cystic epithelium. Despite similar results, in the present sample the percentage of stained cells in each case was higher, probably because of use of the immunohistochemical method. In addition, Lo Muzio et al. (2005) included orthokeratinized cases in their sample.

The results of this study suggest that p63 immunostaining might reflect the immaturity of epithelial cells in this lesion. This aspect may favor tumorigenesis and support the hypothesis of a neoplastic nature of KOTs and of the existence of a suprabasal proliferative compartment as previously described. In addition, the expression of this protein decreases during the differentiation of keratinocytes (Parsa et al. 1999; De Laurenzi et al. 2000;
Morgunkova 2005). According to Yang et al. (1999), $\Delta$ Np63 isoform is capable of inhibiting the wild-type p53 function in a dominant negative manner. Thus, it is possible that p63 plays a role on blocking apoptosis-inducing and growth-inhibitory actions. This may facilitate its proliferative potential on stratified epithelial as described by Yang et al. (1999), De Luca et al. (2006) and Senoo et al. (2007). In addition, p63 contributes to regulation of the Sonic hedgehog [Shh] signaling pathway (Caserta et al. 2006). Alterations in this pathway have been described in different diseases, including KOTs, as mutations in the PTCH gene result in aberrant activation of this pathway (Kochaji et al. 2005). Furthermore, no difference in the expression of Ki-67, p53 or p63 was observed between primary $(n=23)$ and recurrent $(n=14)$ KOTs, in agreement with the findings of $\mathrm{Li}$ et al. (1995) and Lombardi et al. (1995).

The immunohistochemical features of KOTs found in this study suggest the existence of a suprabasal proliferative compartment in the epithelium of such lesions as demonstrated by high levels of Ki-67, p53 and p63. Our results indicate that these proteins contribute to the biologic profile of KOTs, and that p63 plays a role in the regulation of epithelial cell differentiation, and may favor tumorigenesis.

Acknowledgments We thank Ana Cristina Mota, Nancy Silva Santos for excellent technical assistance. This study was supported by CNPq, and grants from FAPESB No. 200/04.

\section{References}

Agaram N, Collins B, Barnes L et al (2004) Molecular analysis to demonstrate that odontogenic keratocysts are neoplastic. Arch Pathol Lab Med 128:313-317

Barbiere C, Pietenpol J (2005) p63 and epithelial biology. Exp Cell Res 312:695-706

Caserta T, Kommagani R, Yuan Z et al (2006) p63 overexpression induces the expression of Sonic Hedgehog. Mol Cancer Res 4:759-768

De Laurenzi V, Rossi A, Terrinoni A et al (2000) p63 and p73 transactivate differentiation gene promoters in human keratinocytes. Biochem Biophys Res 273:342-346

De Luca M, Pellegrini G, Green H (2006) Regeneration of squamous epithelium from stem cells of cultured grafts. Regenerative Med 1:45-57

De Paula A, Carvalhais J, Domingues M et al (2000) Cell proliferation markers in the odontogenic keratocyst. J Oral Pathol Med 29:477-482

Foschini M, Cocchi R, Marucci G et al (2006) High deltaN p63 isoform expression favours recurrences in odontogenic keratocyst-odontogenic keratocyst tumour. Int J Oral Maxilllofac Surg 35:673-675

Gerdes J, Li L, Schlueter L et al (1991) Immunobiochemical and molecular biologic characterization of the cell proliferationassociated nuclear antigen that is defined by monoclonal antibody Ki-67. Am J Pathol 138:867-873 
Giuliani M, Grossi G, Lajolo C et al (2006) Conservative Management of a large Odontogenic Keratocyst: Report of a case and review of the literature. J Oral Maxillofac Surg 64:308-316

Gonzalez-Moles M, Mosqueda-Taylor A, Delgado-Rodriguez M et al (2006) Analysis of p53 protein by PAb240, Ki-67 expression and papillomavirus DNA detection in different types of odontogenic keratocyst. Anticancer Res 26:175-181

Henley J, Summerlin D, Tomich C et al (2005) Molecular evidence supporting the neoplastic nature of odontogenic keratocyst: a laser microdissection study of 15 cases. Histopathology 46: $582-586$

Kaplan I, Hirshberg A (2004) The correlation between epithelial cell proliferation and inflammation in odontogenic keratocyst. Oral Oncol 40:985-991

Kochaji N, Goossens A, Geerts A, Bootenberg P (2005) PTCH expression in odontogenic cysts, a cause of pathogenesis or reason for clinical complication. Oral Oncol 41:284-288

Kolar Z, Geierova M, Bouchal J et al (2006) Immunohistochemical analysis of the biological potential of odontogenic keratocysts. J Oral Pathol Med 35:75-80

Kichi E, Enokiya Y, Muramatsu T et al (2005) Cell proliferation, apoptosis and apoptosis-related factors in odontogenic keratocysts and in dentigerous cysts. J Oral Pathol Med 34:280-286

Kim D, Ahn S, Kim J, Yoon J (2003) Comparative Ki-67 expression and apoptosis in the odontogenic keratocyst associated with or without an impacted tooth in addition to unilocular and multilocular varieties. Yonsei Medical J 44:841-846

Kimi K, Kumamoto H, Ooya K, Motegi K (2000) Immunohistochemical analysis of cell-cycle-and apoptosis-related factors in lining epithelium of odontogenic keratocysts. J Oral Pathol Med 30:434-442

Lane D (1992) Worrying about p53. Curr Biol 2:581-583

Levine A, Momande J, Finlay C (1991) The p53 tumour suppressor gene. Nature 6:453-456

Li TJ, Browne R, Matthews J (1995) Epithelial cell proliferation in odontogenic keratocyst: a comparative immunohistochemical study of ki-67 in simple, recurrent and basal cell nevus syndrome (BCNS)-associated lesions. J Oral Pathol Med 24:221-226

Li TJ, Browne R, Prime $S$ et al (1996) p53 expression in odontogenic keratocyst epithelium. J Oral Pathol Med 25:249-255

Little N, Jochemsen A (2002) p63. Int J Biochem Cell Biol 34:6-9

Lo Muzio L, Staibano S, Pannone G et al (1999) Expression of Cell Cycle and Apoptosis-related Proteins in Sporadic Odontogenic Keratocysts and Odontogenic Keratocysts associated with the Nevoid Basal Cell Carcinoma Syndrome. J Dent Res 78: $1345-1353$

Lo Muzio L, Santarelli R, Caltabiano C et al (2005) p63 expression in odontogenic cysts. Int J Oral Maxillofac Surg 34:668-673

Lombardi T, Odell E, Morgan P (1995) p53 Immunohistochemistry of odontogenic keratocysts in relation to recurrence, basal-cell budding and basal-cell naevus syndrome. Arch Oral Biol 40:1081-1084

McCormick D, Chong H, Hobbs C et al (1993) Detection of the Ki-67 antigen in fixed and wax-embedded sections with the monoclonal antibody MIB1. Histopathology 22:355-360

Mills A (2006) p63: Oncogene or tumor suppressor? Curr Opin Genet Develop 16:1-7

Morgunkova A (2005) The p53 gene family: Control of cell proliferation and development programs. Biochemistry (Moscow) 70:955-971
Nylander K, Coates P, Hall P (2002) Differential expression of p63 isoforms in normal tissue and neoplastic cells. J Pathol 198: 417-427

Oda D, Rivera V, Ghanne N et al (2000) Odontogenic keratocyst: the northwestern USA experience. J Contemp Dental Pract 1:60-74

Ogden G, Chisholm R et al (1992) p53 protein in odontogenic cysts: Increased expression in some odontogenic keratocyst. J Clin Pathol 45:1007-1010

Parsa R, Yang A, McKeon F, Green H (1999) Association of p63 with proliferative potential in normal and neoplastic human keratinocytes. J Invest Dermatol 113:1099-1105

Philipsen H P (2005) Keratocyst odontogenic tumour. In: WHO Classification of tumours. Head and Neck tumours, IARC press, Lyon, pp 306-307

Piattelli A, Foroni M, Santinelli A, Rubini C (2001) p53 expression in odontogenic cysts. J Endodontics 27:459-461

Save V, Nylander K, Hall P (1998) Why is p53 protein stabilized in neoplasia? Some answers but many more questions! J Pathol 184:348-350

Senoo M, Pinto F, Crum C, McKeon F (2007) p63 is essential for the proliferative potential of stem cells in stratified epithelia. Cell 129:523-536

Shear M (2002a) The aggressive nature of the odontogenic keratocyst: is it a benign cystic neoplasm? Part 1 Clinical and early experimental evidence of aggressive behaviour. Oral Oncol $38: 219-226$

Shear M (2002b) The aggressive nature of the odontogenic keratocyst: is it a benign cystic neoplasm? Part 2 Proliferation and genetic studies. Oral Oncol 38:323-331

Shear M (2002c) The aggressive nature of the odontogenic keratocyst: is it a benign cystic neoplasm? Part 3 Immunocytochemistry of cytokeratin and other epithelial cell markers. Oral Oncol 38: 407-415

Slootweg P (1995) p53 and Ki-67 reactivity in epithelial odontogenic lesion. An Immunohistochemical study. J Oral Pathol Med 24:393-397

Stoelinga P (2001) Long-term follow-up on keratocysts treated according to a defined protocol. Int $\mathrm{J}$ Oral Maxillofac Surg 30:14-25

Thosaporn W, Iamaroon A, Pongsiriwet S, Ng K (2004) A comparative study of epithelial cell proliferation between the odontogenic keratocyst, orthokeratinized odontogenic cyst, dentigerous cyst and ameloblastoma. Oral Dis 10:22-26

Verheijen R, Kuijpers H, Schlingemman R et al (1989) Ki-67 detects a nuclear matrix-associated proliferation-related antigen. I. Intracellular localization during interphase. J Cell Science 92:123-130

Yang A, Kaghad M, Wang Y et al (1998) p63, a p53 homolog at 3q27-29, encodes multiple products with transactivating, deathinducing, and dominant-negative activities. Mol Cell 2:305-316

Yang A, Kaghad M, Wang Y et al (1999) p63 is essential for regenerative proliferation in limb, craniofacial and epithelial development. Nature 398:714-718

Yang A, Kaghad M, Caput D, McKeon F (2002) On the shoulders of giants: p63, p73 and the rise of p53. Trends Genet 8:90-95 